\title{
A case of left orbital cysticercosis presenting as acute preseptal cellulitis.
}

\author{
Mithun Thulasidas*, Vasanthi Kotian, Abin Holla, Sumitha CV \\ Department of Ophthalmology, K.S Hegde Charitable Hospital, Nithyanandanagar, Deralakatte, Mangalore, \\ India
}

\begin{abstract}
Purpose: The purpose is to report a case of left orbital cysticercosis, presented with signs of acute preseptal cellulitis.

Case report: Orbital infection is a form of inflammation caused by infective agents and, therefore, orbital infection and other orbital inflammatory processes can have similar presentation. Parasitic infestations of the orbit are rare, commonly located in the superolateral and superomedial angles of the orbit. We report a case of 31 year old man who presented with acute painful rapidly progressive periocular swelling, didn't show any response to 1 week of systemic antibiotics. Left orbital cyst excision was done after correlating with radiological findings and histopathological features were suggestive of parasitic infected cyst.

Conclusion: Parasitic infected cyst could be considered in the differential diagnosis of unilateral periocular swelling with proptosis, commonly in superior part.
\end{abstract}

Keywords: Intraorbital parasitic cyst, Preseptal cellulitis, Proptosis.

Accepted on April 20, 2018

\section{Introduction}

Orbital infection is a form of inflammation caused by infective agents and, therefore, orbital infection and other orbital inflammatory processes can have similar presentation [1]. Patients with atypical presentation or those who are unresponsive to medical treatment should have an orbital biopsy for pathological diagnosis [2]. Parasitic infestations of the orbit are rare [1], commonly located in the superolateral and superomedial angles of the orbit. Cysticercosis is caused by infection with intermediate larval stage of Taenia solium. Human beings are definitive hosts that harbor the adult parasite in the intestine and pigs are the intermediate hosts harboring the larvae. Human cysticercosis occurs when they act as intermediate host by ingesting the eggs via following modes of infestation: a) contaminated food and water with the Taenia solium eggs (hetero-infection); b) reinfection by ingestion ova of the existing parasite (external auto-infection); c) retrograde peristalsis causing the transport of mature proglottids bearing eggs from bowel to stomach (internal auto-infection) [3]. The most common clinical findings are periocular swelling, proptosis, ptosis and restriction of ocular motility [4]. We present a case of left orbital cysticercosis presented with signs of acute preseptal cellulitis

\section{Case Report}

A 31 year old man presented with painful rapidly progressive periocular swelling since one week. There was no history of trauma or systemic disease. He is a vegetearian. He had a visual acuity of $6 / 6$ in both eyes with normal head posture, orthophoric gaze and full range ocular movements. Ocular examination showed maceration of eyelids, mild axial proptosis (exophthalmometry $\sim 23 \mathrm{~mm}$ ) and mechanical ptosis (Figure 1). The mass was tender, warm, irreducible, non pulsatile, without any bruit and was felt in the superior part of the orbit; posterior limit could not be reached. Fundus was normal in both eyes.

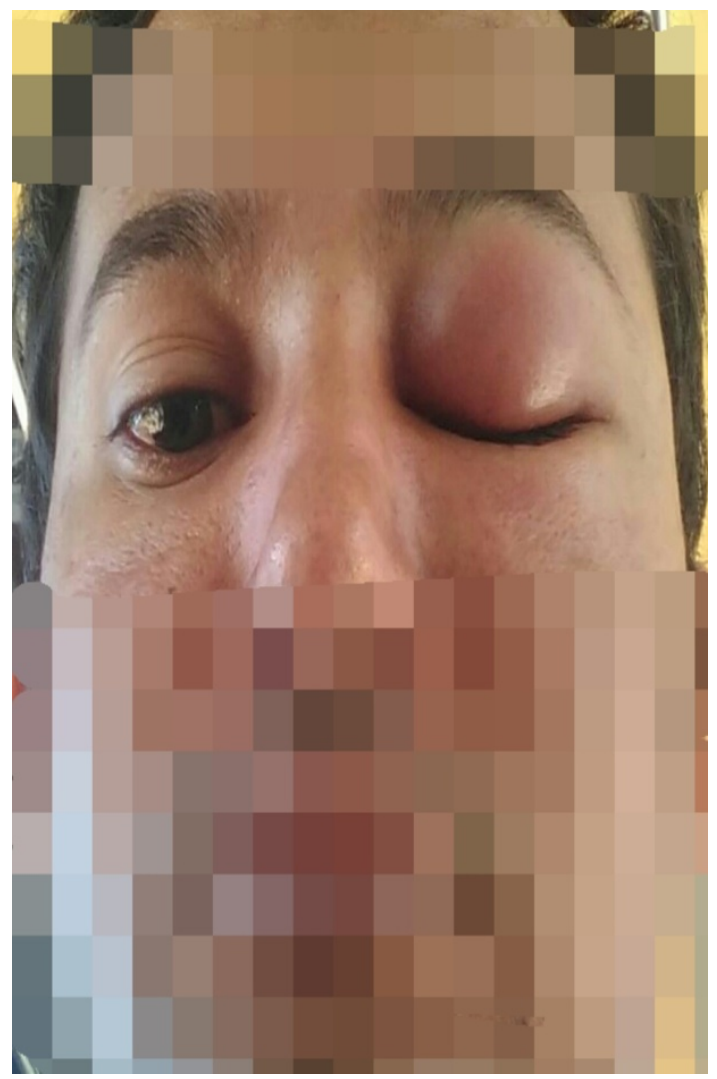

Figure 1: showing left mild axial proptosis with mechanical ptosis.

Patient was treated on systemic antibiotics and non-steroidal anti-inflammatory drugs for one week. Inflammation was controlled but the swelling didn't respond to antibiotics. 
A magnetic resonance imaging (MRI) revealed a hypodense lesion located in the superior part of the orbit (Figures $2 \mathrm{a}$ and 2b).

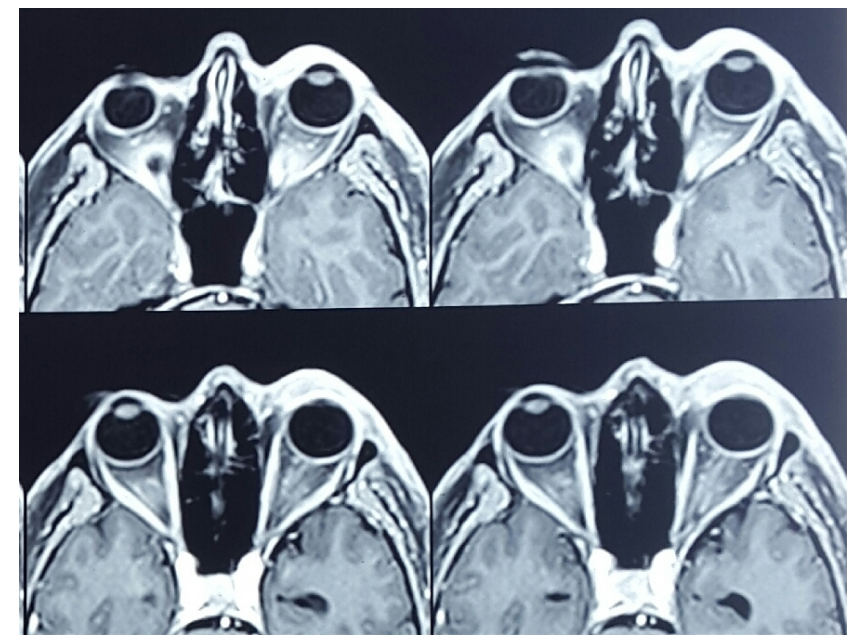

Figure 2a: Axial section MRI sowing left orbit hypodense lesion.

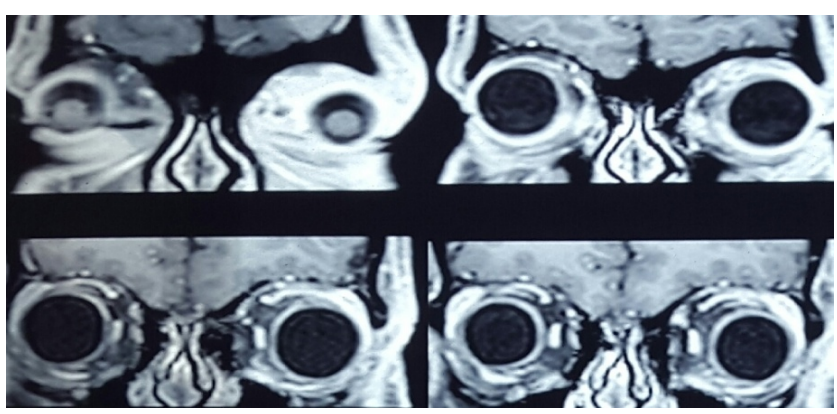

Figure 2b: Coronal section MRI showing left orbit hypodense lesion.

Left orbit cyst excision was done using transcutaneous approach after correlating with radiological findings. Sample was sent for histopathology analysis (Figure 3).

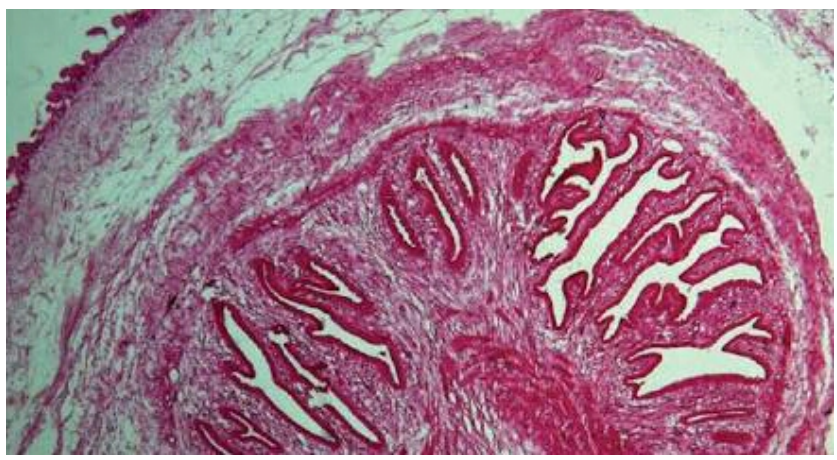

Figure 3: Histopathologic appearance of cysticercosis showing scolex with sucker (H\&E 40x).

Histopathologic features showing scolex with sucker and hooklets surrounded by a well-defined cyst wall were suggestive of Parasitic infected cyst (Cysticercosis).

ELISA for cysticercosis was negative.

MRI brain and USG abdomen were normal.
After one month of oral Albendazole $400 \mathrm{mg} \mathrm{BD}$, the patient was doing well and no proptosis or enophthalmos detected (Figure 4).

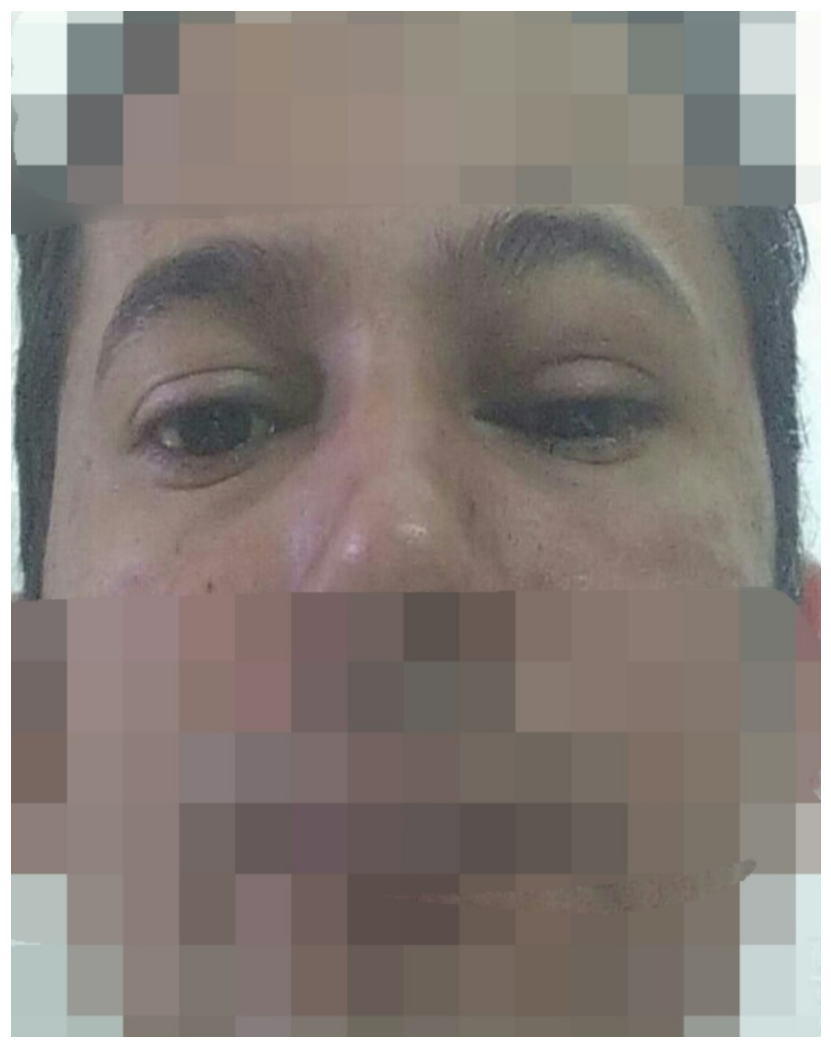

Figure 4: After 1 month of oral antihelminthics.

\section{Discussion}

Parasitic infestations of the orbit are rare and have highest prevalence in developing countries [1] Cysticercosis is a common parasitic infestation worldwide including India and can affect almost all eye tissues like the vitreous cavity, subretinal space, subconjunctival tissue, orbit and lid. Cysticercus cellulosae can be a cause of orbital cellulitis also [1]. Atul et al., who studied the sociodemographic trends in ocular cysticercosis in India, showed a male preponderance of $2: 1$, with the maximum number of patients seen in the fourth decade; $30 \%$ of them were vegetarians [5]. Diagnosis of cysticercosis requires radiological visualisation using MRI or CT. ELISA for cysticercosis can be supportive evidence for diagnosis but can be negative. Histopathology is the only confirmatory feature in those cases not responding to antibiotics and with normal eosinophil count. Neurological examination is mandatory after the diagnosis of cysticercosis. Neurocysticercosis was found to be the most common cause of epilepsy in a study from South India [6]. Treatment should include total surgical excision of the cyst without rupture to avoid relapse and starting on oral antihelminthics for almost 1 month [7]. Patients and their family members should be educated to improve sanitation and food-preparation hygiene in endemic areas. Parasitic infected cyst could be considered in the differential diagnosis of unilateral periocular swelling with proptosis, commonly in superior part. 
Citation: Mithun T, Vasanthi K, Abin H, et al. A case of left orbital cysticercosis presenting as acute preseptal cellulitis. J Clin Ophthalmol. 2018;2(1):60-62.

\section{References}

1. Vincent B Lam Choi, Hunter K L Yuen, Jyotirmay Biswas, et al. Update in Pathological Diagnosis of Orbital Infections and Inflammations. Middle East Afr J Ophthalmol. 2011;18(4):268-76.

2. Yuen SJ, Rubin PA. Idiopathic orbital inflammation: distribution, clinical features, and treatment outcome. Arch Ophthalmol.2003;121(4):491-9.

3. Dhiman R, Devi S, Duraipandi K, et al. Cysticercosis of the eye. Int J Ophthalmol. 2017;10(8):1319-24.

4. Rath S, Honavar SG, Naik M, et al. Orbital cysticercosis: clinical manifestations, diagnosis, management, and outcome. Ophthalmology. 2010;117:600-5.

5. Atul K, Kumar TH, Mallika G, et al. Socio-demographic trends in ocular cysticercosis.Acta Ophthalmol Scand. 1995;73:438-41.

6. World Health Organization Control of neurocysticercosis, Report by Secretariat, 55th World Health Assembly Provisional agenda item 13.18 A55/23, 5 April 2002.
7. Subramanyam M. Surgical atlas of orbital diseases. $2008 ; 212$

\section{*Correspondence to}

Dr. Mithun Thulasidas

Junior resident, Department of Ophthalmology

K.S Hegde Charitable Hospital

Nithyanandanagar, Deralakatte

Mangalore- 575018

Tel: 7406298745

E-mail: mithun.thulasidas@gmail.com 